\title{
SUICIDAL THOUGHTS AS COGNITIVE RISK FACTORS FOR SUICIDE AMONG CANCER PATIENTS
}

\author{
Dr. Lenče Miloševa, Faculty of Medical Science, Goce Delčev University, Štip, Macedonia \\ E-mail: lence.miloseva@ugd.edu.mk \\ Dr. Vladimir Milošev, Clinical Hospital, Štip, Macedonia \\ E-mail: vlmilos@gmail.com \\ Dr. Roza Arsova, Clinical Hospital, Štip, Macedonia \\ E-mail: rozaberkova@yahoo.com \\ Dr. Svetlana Jovevska, Faculty of Medical Science, Goce Delčev University, Štip, Macedonia \\ E-mail: svetlana.jovevska@ugd.edu.mk
}

\author{
A R T I C L E I N F O \\ Original Article \\ Received: October, 29.2016. \\ Revised: November, 28.2016. \\ Accepted: December, 01.2016. \\ doi:10.5937/IJCRSEE1602053M \\ UDK \\ 616.89-008.441.44- \\ 056.24(497.7)"2014/2016”
}

\section{Keywords:}

suicidal thoughts,

depression,

cognitions,

risk factors,

cancer.

\begin{abstract}
A B S T R A C T
The aim of this study is twofold. The first aim is to investigate whether we can use the Patient Health Questionnaire-9 (PHQ-9), translated on Macedonian language, item 9 in order to screen passive suicidal thoughts. The second aim is to describe the nature of ,better to be death thoughts or hurting own self in some way" reported with structure clinical interviews carried out in order to further asses passive suicidal thoughts of cancer patients, who hade endorsed item 9 of this scale. The data were collected from Department of maxillofacial surgery, Clinical Hospital, Stip, Macedonia, during the period june 2014 - june 2016. The final survey sample consists of 122 patients ( $66 \%$ female and $34 \%$ male) aged $19-70$ years. Inclusive criteria: patients were diagnosed with cancer in maxillofacial region. We applied Patient Health Questionnaire-9 (PHQ-9) and structured clinical interview. Two weeks after they become familiar with the diagnose they filled up PHQ-9 scale, and after four weeks clinical interviews were carried out. The reliability (Cronbach`s alpha) for the PHQ-9 scale translated on Macedonian language and sample was good (alpha $>0.85$ ). We found that patients with diagnosed cancer who endorsed ,passive suicide ideas item 9 “" were more likely to have suicidal thoughts which were described latter on during clinical interview. We analyzed thoughts reported by patients in the frame of modified categories of suicidality. Of 67 (54.92\%) of patients who endorsed item 9 with ,yes“, the highest percentige was in category with passive suicidal thoughts $39(58.21 \%)$. Due to the limitation of this study, more studies are needed in future.
\end{abstract}

(C) 2016 IJCRSEE. All rights reserved.

\section{INTRODUCTION}

Despite serious concerns about the risk of suicide among patients diagnosed with cancer in clinical practice, the analysis of literature and previous studies worldwide shows that there are only few studies that treat this issue (Akechi,Okamura, Nishiwaki, \& Uchitomi, 2002; Misono, Weiss, Fann, Redman, \&

Corresponding Author

Dr. Lenče Miloševa, Faculty of Medical Science, Goce Delčev University, Stip, Macedonia

E-mail: lence.miloseva@ugd.edu.mk

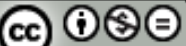

This work is licensed under a Creative Commons Attribution - NonCommercial - NoDerivs 4.0. The article is published with Open Access at www.ijcrsee.com
Yueh, 2008; Walker, Hansen, Buthcher, Sharma, Wall, Murray, \& Sharpe, 2011; Robson, Scrutton, Wilkinson, \& MacLeod, 2010). One of the basic tenets of the cognitive behavioral model of suicide explanation from which we start, is that having suicidal thoughts lead to action, then better conceptual understanding of suicidal ideas can offer clarification on how an individual will act (or not) according to these ideas. Screening of clinically relevant risk factors for suicide and identifying specific differences between passive and active suicidal ideation allow clinicians to identify the group of individuals at risk of suicide, to offer appropriate prevention and effective treatment.

In recent years, researchers and clinicians do not treat passive suicidal thoughts as 
clinically relevant risk factor for suicide, while underestimating the strength of this desire to die, compared with making a plan for suicide in individuals having active suicidal ideation. Identification of passive suicidal thoughts as a clinical relevant risk factor contributes to shedding light on the etiological picture of clinical depression and suicide phenomena among cancer patients. Passive suicidal ideation can be characterized as emotionally colored thoughts, more as a wish, according to which life is not worth living or that it is better for the person to be dead (Moran, 2013). One third of individuals with suicidal attempts in the past had passive suicidal ideation, or had plans for committing suicide (Baca-Garcia, Perez-Rodriguez, Oquendo, Keyes, Hasin, Grant, \& Blanco, 2011; Moran, 2013).

The aim of this study is twofold. The first aim is to investigate whether we can use the Patient Health Questionnaire-9 (PHQ-9, Kroenke, Spitzer, \& Williams, 2001), translated on Macedonian language, item 9 in order to screen passive suicidal thoughts. The second aim is to describe the nature of ,,better to be death thoughts or hurting own self in some way" reported with structure clinical interviews carried out in order to further asses passive suicidal thoughts of participants, cancer patients, who hade endorsed item 9 of this scale.

\section{MATERIALS AND METHODS}

In the frame of the „Goce Delcev“ University, Stip, supported project, the data were collected from Department of maxillofacial surgery, Clinical Hospital, Stip, Macedonia, during the period June 2014 - June 2016. The study was approved by the Ethics Committee of the Goce Delcev" University, Stip.

The final survey sample consisted of 122 patients (66\% female and 34\% male) aged 19 70 years. Inclusive criteria: patients were diagnosed with cancer in maxillofacial region. We applied Patient Health Questionnaire-9 (PHQ-9, Kroenke, Spitzer, \& Williams, 2001), translated on Macedonian language and structured clinical interview. The Patient Health Questionnaire-9 (PHQ-9), is a well known a self report scale comprised of nine items developed to measure depression. For each item the patients are asked to asses how much they were bothered by the symptoms over the last two weeks. Each of the nine items is scored from zero to three, which results in a maximum summed score of 27 , indicated the de- gree of depression. Item 9 asks, „,Over the last two weeks how often have you been bothered by the following problem: thoughts that you would be better off dead, or of hurting yourself in some way"? (Kroenke, Spitzer, \& Williams, 2001). There are four answer options: „not at all“" (scoring zero), "several days“" (scoring one), ,more than half the days" (scoring two) or ,nearly every day“ (scoring three).

Those patients who reported such thoughts for at least several days in that given period were labeled positive responders. Two weeks after they become familiar with the diagnose they filled up PHQ-9 scale, and after four weeks clinical interviews were carried out. Written informed consent was obtained by all study participants.

\section{RESULTS}

First of all we checked the reliability (Cronbach`s alpha) for the PHQ-9 scale translated on Macedonian language and sample. The reliability coefficient was good (alpha> 0.85 ). Than we applied PHQ-9 scale. Overall, $67(54.92 \%)$ of 122 of patients with diagnosed cancer in maxillofacial region were positive responders to Item 9 of the PHQ-9, which indicated that they had thoughts that they would be better off dead or thoughts of hurting themselves for at least several days in the preceding 2 weeks. We found that patients with diagnosed cancer who endorsed ,passive suicide ideas item 9" were more likely to have suicidal thoughts which were described later during structured clinical interview.

Based on written notes from a structured clinical interview conducted with each patient, based on consensus, the authors of this research placed each patient's response in the proposed categories. We analyzed thoughts reported by patients in the frame of modified categories of suicidality based on Walker's research (Walker, Hansen, Buthcher, Sharma, Wall, Murray, \& Sharpe, 2011). It is about six clinically significant categories for which consensus was reached between the authors of the paper (Table 1). The next step was to calculate the number and proportion of patients in each of the categories. Table 1 shows the number and percentage of patients placed in each categories of suicidality. Table 2 shows examples responses at interview. 
Table 1. Categories of Reported Thoughts of Death and Suicide over preceding 4 weeks as elicited at interview and the number $(\%)$ of patients reporting each.

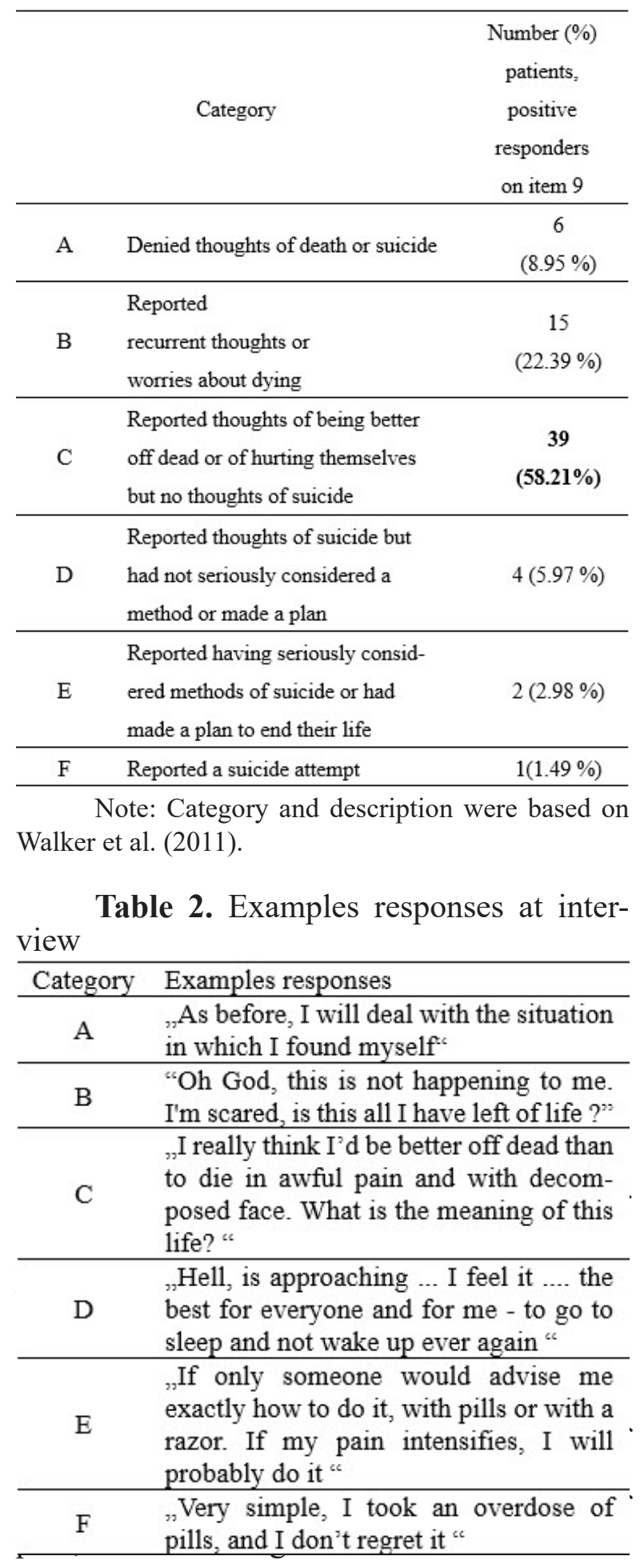

\section{DISCUSSIONS}

The analysis of the results obtained showed that $8.95 \%$ of positive respondents on item 9, denied any thoughts that they would be better off dead (category A) and $22.39 \%$ reported recurrent thoughts or worries about dying (category B).

Of $67(54.92 \%)$ of patients who endorsed item 9 with ,yes“, the highest percentige was in category with passive suicidal thoughts $58.21 \%$. With other words, the greatest percentage of positive respondents is precisely in category $\mathrm{C}$. They conveyed their thoughts that they would be better off dead, but not that they tried it or committed suicide. $5.97 \%$ of respondents (D) are without a specific plan or method, but with the presence of thoughts of suicide. The remaining $2.98 \%$ and $1.49 \%$ of positive responders (category $\mathrm{E}$ and $\mathrm{F}$, respectively) reported serious and clear thoughts of committing suicide.

We made a comparison of the data obtained with those from Walker's research (2011), which was the starting base for conducting our research. There are differences in the proportion of respondents in the categories, as well as similarities in terms of the detailed description of the thoughts about death and suicide and the finding that patients with a higher score of item- 9 are more inclined to have and communicate suicidal thoughts.

To our knowledge, in the Republic of Macedonia such a research among cancer patients in maxillofacial region has not been conducted so far. The research has three limitations. The first relates to the generalizing of the findings. The survey was conducted in one clinic only and with patients diagnosed with cancer in maxillofacial region, and therefore we cannot make conclusions about findings typical of patients with other diagnosed cancers. The second limitation relates to the application of selfreporting tests (PHQ-9) and structured clinical interview, which rely solely on the patient. The third limitation concerns the fact that many other important variables should be included in this kind of research, such as the patients' general health and their social support.

\section{CONCLUSIONS}

We can conclude that patients with serious diseases such as cancer are at higher risk of suicide (Misono, Weiss, Fann, Redman, \& Yueh, 2008; Robson, Scrutton, Wilkinson, \& MacLeod, 2010). The interpretation and comparison of findings of such studies is complicated because patients suffering from diseases that threaten their life are more preoccupied with thoughts of death than of suicide. We responded to the first objective of the research 
- that we can use the PHQ-9 for screening passive suicidal ideation, but an obligatory and clinical interview is necessary in order to know which patients are at risk of suicide. We also achieved the second objective set in the research, and that is the answer to the question what patients actually think when they respond positively to item 9 .

Methodological difficulties and the small number of conducted surveys make it difficult to identify risk factors and causes of suicide among cancer patients. Although significant progress in improving the quality of life of cancer patients has been made in the world, this research tells us that a significant number of patients communicate thoughts of being better off dead or thoughts of hurting themselves. These are alarming findings that oblige us to conduct further research.

There is a need of multidisciplinary work and teams between maxillofacial surgeons, clinical psychologist, psychiatrist and oncologist. We consider the follow up studies and the involvement of more important variables in research to be of great importance for determining the predictive risk factors for suicide among patients with cancer. We believe that this research has applicative value and that it has not only theoretical and empirical contributions, but has contributions to clinical practice as well because patients with severe medical illnesses such as cancer are at increased risk of suicide (Misono, Weiss, Fann, Redman, \& Yueh, 2008).

\section{ACKNOWLEDGEMENTS}

This paper is supported and is the part of Goce Delcev University project. We would like to express our gratitude for this support and for collaboration with colleagues from Clinical Hospital, Dept. of Maxillofacial surgery and Dept. of Psychiatry.

\section{Conflict of interests}

The authors declare no conflict of interest.

\section{REFERENCES}

Akechi, T., Okamura, H., Nishiwaki, Y., \& Uchitomi, Y. (2002). Predictive factors for suicidal ideation in patients with unresectable lung carcinoma. Cancer, 95(5), 1085-1093. doi:10.1002/cncr.10769

Baca-Garcia, E., Perez-Rodriguez, M. M., Oquendo, M. A., Keyes, K. M., Hasin, D. S., Grant, B. F., \& Blanco, C. (2011). Estimating risk for suicide attempt: Are we asking the right questions?: Passive suicidal ideation as a marker for suicidal behavior. Journal of affective disorders, 134(1), 327-332. doi: 10.1016/j.jad.2011.06.026

Kessler, R. C., Borges, G., \& Walters, E. E. (1999). Prevalence of and risk factors for lifetime suicide attempts in the National Comorbidity Survey. Archives of general psychiatry, 56(7), 617626.

Kroenke, K., Spitzer,R.L., \& Williams, J.B.W. (2001). The PHQ-9: Validity of a Brief Depression Severity Measure. Journal of General Internal Medicine, 16(9), 606-613. http://doi. org/10.1046/j.1525-1497.2001.016009606.x

Misono, S., Weiss, N. S., Fann, J. R., Redman, M., \& Yueh, B. (2008). Incidence of suicide in persons with cancer. Journal of Clinical Oncology, 26(29), 4731-4738. http://doi.org/10.1200/ JCO.2007.13.8941

Moran, C. (2013). Passive Suicidal Ideation: A Clinically Relevant Risk Factor for Suicide (Doctoral dissertation, Case Western Reserve University).

Robson, A., Scrutton, F., Wilkinson, L., \& MacLeod, F. (2010). The risk of suicide in cancer patients: a review of the literature. Psycho-Oncology, $19,1250-1258$.

Walker, J., Hansen, C. H., Butcher, I., Sharma, N., Wall, L., Murray, G., \& Sharpe, M. (2011). Thoughts of death and suicide reported by cancer patients who endorsed the "suicidal thoughts" item of the PHQ-9 during routine screening for depression. Psychosomatics, 52(5), 424-427. http://dx.doi. org/10.1016/j.psym.2011.02.003 